\title{
Functional Readings and Wide-Scope Indefinites
}

\author{
Yoad Winter \\ Technion/UiL OTS
}

\section{Introduction}

Traditional formal semantics assumes that quantification in natural language operates only on atomic entities. However, much recent work in natural language semanties has shown the advantages of more complex forms of quantification that involve functions over atomic domains. Two areas of functional quantification have received special attention. One area concerns the so-called functional and pair-list interpretation of questions and copular sentences. Another area deals with the wide scope interpretation of indefinite NPs. Quantification over Skolem functions is used to model both kinds of phenomena. Some theories restrict the usage of Skolem functions to the simple case of choice functions: Skolem functions that map any non-empty set to an entity in this set.

Despite the similarity in the mechanisms that are used for describing functional readings and wide scope indefinites, no attempt has so far been made to bring them into one framework. This paper proposes such a unified mechanism, which is based on a similar type shifting operator to the one proposed by Jacobson (these proceedings). Like Jacobson's operator, the proposed mapping establishes a connection between functions from entities to entities on the one hand, and binary relations over entities, on the other hand. The mechanism accounts for the similar distribution of functional readings and wide-scope readings beyond islands with different NPs, and leads to a novel hypothesis about the class of quantifiers that license functional interpretations and the reasons for their restricted distribution.

The structure of this paper is as follows. Section 2 briefly overviews the problems of functional readings and wide scope indefinites, gives necessary technical details about previous approaches and discusses the motivation for a unified analysis. Section 3 introduces the mapping that allows an extended theory to treat both phenomena and exemplifies its applications. Section 4 is a short note on the relations between Jacobson (these proceedings) and the present proposal.

This paper is an abridged version of an unpublished work in preparation that was prepublished as Winter (2001b) and is available electronically.

\section{Functional readings and wide scope indefinites}

\subsection{Functional readings}

The so-called functional reading of questions can be illustrated by the following familiar question-answer pairs. 
(1) a. Which woman does every man love? His mother.

b. Which woman does no man love? His mother-in-law.

The problem of interpreting questions that exhibit this kind of reading was discussed extensively in the literature. ${ }^{1}$ The puzzle is often related (cf. Chierchia 1993) to the problem of pair-list readings of questions, as illustrated by the following short discourse.

(2) Which woman does every man love? John loves Mary, Bill loves Sue, etc.

In order to analyze both kinds of readings that questions exhibit, it has been proposed that quantification over functions plays a role in the interpretative procedure. Similar mechanisms have been proposed for the functional readings of copular sentences like the following.

a. The woman that every man loves is his mother.

b. The woman that no man loves is his mother-in-law.

Sharvit (1999) convincingly argues that such copular sentences have the same distribution and syntactic/semantic properties of functional questions as in (1). In order to illustrate the mechanisms that will be considered, we will therefore concentrate on such indicative copular sentences, without getting into the more intricate semantics of questions.

A fully worked-out account of functional readings in copular sentences as in (3) is given in Jacobson (1994). Jacobson's analysis is based on her general theory of variable-free semantics for anaphora. The theory itself is introduced in much detail in Jacobson $(1999,2000)$, and I will not try to review all of its parts here. Jacobson's assumptions that are important for our present purposes are the following, which for convenience are given the names (J1)-(J4).

(J1) An expression $E$ that contains a "free pronoun" $P$ denotes a function from entities of the standard type of $P$ to entities of the standard type of $E$.

Consider for instance the noun phrase the woman who gave him birth or, equivalently his mother (as in (3)). Assume that the standard type of NPs is $e$ and that this is also the standard type of the pronoun him. Jacobson therefore assumes that the whole NP denotes a function of type ee: a function from entities to entities. In the example, this is the function that maps every (male human) entity to its mother.

(J2) Transitive predicates like love, of the standard type $e(e t)$, have a secondary meaning of type $(e e)(e t)$ that ranges over ee functions in the object argument. This reading enables the subject NP to "bind" a pronoun within the object. The operator that derives this additional meaning of transitive predicates is denoted ' $\mathrm{Z}$ ' and is defined as follows.

(4) $\mathrm{Z}_{(e(e t))((e e)(e t))} \stackrel{\text { def }}{=} \lambda R_{e(e t)} \cdot \lambda f_{e e} \cdot \lambda x_{e} \cdot R(f(x))(x)$ 
In words: the $\mathrm{Z}$ function maps a binary relation $R$ to the relation $\mathrm{Z}(R)$ that holds exactly between those ee-type functions $f$ and entities $x$ that satisfy $R(f(x))(x)$.

For instance, the following example (5a) is analyzed as in (5b).

a. Every man loves his mother.

b. $\operatorname{every}_{(e t)((e t) t)}^{\prime}\left(\operatorname{man}_{e t}^{\prime}\right)\left(\mathrm{Z}\left(\right.\right.$ love $\left._{e(e t)}^{\prime}\right)\left(\right.$ his_mother $\left.\left.{ }_{e e}^{\prime}\right)\right)$ $\Leftrightarrow \operatorname{every}^{\prime}\left(\operatorname{man}^{\prime}\right)\left(\left\{x:\right.\right.$ love' $\left(\right.$ his $\left.\left.\left.\_\operatorname{mother}^{\prime}(x)\right)(x)\right\}\right)$ $\Leftrightarrow \operatorname{man}^{\prime} \subseteq\left\{x: \operatorname{love}^{\prime}\left(\right.\right.$ his_mother $\left.\left.{ }^{\prime}(x)\right)(x)\right\}$

The determiner every here standardly denotes the subset relation between sets.

(J3) Items like the definite article the, the relative pronoun that and the copula be can range over ee functions as well as "ordinary" $e$-type entities. In essence, we can assume that such items denote the (polymorphic) iota, intersection and identity functions respectively.

(J4) Intransitive nouns like woman, of the standard type et, have a second meaning of type $(e e) t$. This meaning ranges over ee functions and allows the noun to combine with functional relative clauses. I use ' $\mathrm{N}$ ' to denote the operator that derives this additional meaning of transitive predicates. This operator is defined as follows.

$$
\mathrm{N}_{(e t)((e e) t)} \stackrel{\text { def }}{=} \lambda P_{e t} \cdot \lambda f_{e e} \cdot \forall x_{e}\{P(f(x))\}
$$

For instance, the $(e e) t$ denotation of the noun woman that the $\mathrm{N}$ operator derives is the set of $e e$ functions that map each entity to a woman. Note that this set is empty when there are no women in the model.

For sake of exposition, I will use here a slightly modified version of the $\mathrm{Z}$ operator that Jacobson uses for binding. This revised operator, which is denoted ' $\mathrm{Z}^{0}$ ', allows generalized quantifiers of type (et) $t$, rather than $e$ type entities, to combine directly with the binary relation that is modified by the operator. Its definition follows.

(7) $\mathrm{Z}_{(e(e t))(((e t) t)((e e) t))} \stackrel{\text { def }}{=} \lambda R_{e(e t)} \cdot \lambda Q_{(e t) t} \cdot \lambda f_{e e} \cdot Q\left(\lambda x_{e} \cdot R(f(x))(x)\right)$

This operator has essentially the same consequences of Jacobson's Z operator, but its arguments are now a quantifier and an ee function (in this order), instead of an $e e$ function and an entity as in Jacobson's analysis. This revised formulation of Z only comes to allow a generalized quantifier such as every man in the relative clause that every man loves to "saturate" the subject argument of the transitive predicate loves, without getting into complex questions concerning the derivation of this option within a general categorial theory. ${ }^{2}$

Now we can get back to the sentences in (3) and illustrate their analysis in Jacobson's approach. A derivation of the meaning of sentence (3a) is summarized 
in figure 1 at the end of the article. The type variable $\tau$ stands for any monomorphic type. It is not hard to verify that the result of the above derivation is tantamount to the following statement:

(8) There is only one ee function $f$ s.t. $f$ maps every man to a woman he loves, and this function is the function that maps every man to his mother.

This paraphrase captures the intuitive meaning of sentence (3a). Furthermore, this meaning is derived without any scope shifting of the noun phrase every man beyond the complex NP island, which would have been problematic (for arguments why, see Jacobson (1994) and Sharvit (1999), among others).

Alexopoulou and Heycock (2001) observe that copular sentences exhibit functional readings also in cases where their subject is not necessarily a singular definite as in (3). Some examples for this observation follow.

(9) a. One/a (certain) woman that every man loves is his mother.

b. One/a (certain) woman that no man loves is his mother-in-law.

(10) A woman that every/no man would be happy to see again is his childhood sweetheart.

(11) a. (The) two women that every Frenchman admires are his mother and Brigitte Bardot. (after Engdahl (1986:ch.4))

b. (The) two women that no Frenchman admires are his mother-in-law and Margaret Thatcher.

Jacobson does not mention such sentences, and their functional readings are not immediately captured by her mechanism. However, along the lines of her proposal, it is natural to assume that pre-nominal items such as one, $a$ and (the) two should also be analyzed as polymorphic operators, similar to the polymorphic iota operator that Jacobson assigns to the definite article. For instance, the items one and $a$ in (9) and (10) could be analyzed as polymorphic existential determiners, of type $(\tau t)((\tau t) t)$. When $\tau$ is the ee type, of functions from entities to entities, this would allow the determiner to compose with a restricting predicate of type $(e e) t$ as in Jacobson's polymorphic analysis of the definite article.

Despite its plausibility, such an analysis would be unnecessarily strong, because other NPs do not lend themselves so easily to functional readings. Consider for instance the following unacceptable sentences.

(12) ??At most/at least one woman that every man loves is his mother.

(13) ??No woman that every man would be happy to see again is his childhood sweetheart.

(14) ??Between two and three women that every Frenchman admires are his mother, Brigitte Bardot and possibly Isabelle Adjani.

In (14), for instance, it could be expected that the sentence would entail that there are between two and three functions that send each Frenchman to a woman he admires, in a similar way to the entailment from (11a) to the existence of two such 
functions. However, sentence (14) is quite incoherent. Thus, it would not be too helpful to assign a polymorphic meaning to all the determiners in natural language, since we only need a proper subset of them (if any) to range over functions. In this paper I propose that in fact, no determiner should range over functions. According to the proposed mechanism, functional quantification is only existential and it is derived by the same general functional process that is responsible for the interpretation of wide scope indefinites.

\subsection{Wide-scope indefinites}

The main reason for introducing functions in the semantic analysis of indefinites is their ability to take wide scope (WS) beyond syntactic islands, which has received much attention in the literature since Fodor and Sag (1982). Consider for instance the following example.

(15) If some/a (certain) girl I know arrives to the party then John will be happy.

This sentence has a reading that can be paraphrased as making a statement about a "specific girl", whose arrival to the party will please John.

In recent years, many authors have followed Reinhart $(1992,1997)$ and Kratzer (1998), and assumed that WS indefinites should be treated using choice functions (CFs), whose definition is given below.

Definition 1 Let $E$ be a non-empty set. A function from $\wp(E)$ to $E$ is a choice function over $E$ iff for every $A \subseteq E$ : if $A$ is not empty then $f(A) \in A$.

In extensional type logical frameworks like the one assumed throughout this paper, the set $C H^{\tau}$ of choice functions over type $\tau$ is defined as follows:

$$
C H^{\tau} \stackrel{\text { def }}{=} \lambda f_{(\tau t) \tau} . \forall P_{\tau t} \neq \emptyset[P(f(P))]
$$

Convention: we often write ' $C H$ ' instead of ' $C H^{e}$ ', for the CFs over the domain of entities.

The WS behavior of indefinites is treated using CFs by letting a free CF variable apply to the restriction of the indefinite. We assume that an existential quantifier applies to this variable at the matrix level, and derives the following interpretation of sentence $(15) .^{3}$

$$
\text { (17) } \exists f\left[C H(f) \wedge\left[\operatorname{arrive}^{\prime}\left(f\left(\operatorname{girl}^{\prime}\right)\right) \rightarrow \operatorname{glad}^{\prime}\left(\mathbf{j}^{\prime}\right)\right]\right]
$$

Although indefinites with some and $a$ (certain) show WS behavior beyond islands, many other NPs (e.g. universal NPs) do not show this island-free behavior. It is therefore important to characterize those NPs to which the CF mechanism applies. This question is taken up in Winter (2001a:ch.4), where it is argued that in addition to simple indefinites as in (15), the CF mechanism also applies to simple numeral indefinites (e.g. three students), $W H$ phrases and singular and plural definite NPs. The WS potential of simple numerals can be easily illustrated by the interpretation (18b) of sentence (18a).

(18) a. If three girls I know arrive to the party then John will be happy. 
b. There is a set $A$ of three girls that I know such that if the girls in $A$ arrive to the party then John will be happy.

The WS potential of WH elements is discussed (among others) by Reinhart (1997), using question-answer pairs like the following:

(19) Who will be offended if we invite which philosopher? John will be offended if we invite Putnam.

To interpret the noun phrase which philoso pher, early semantic theories of questions would have to assign it sentential scope over the conditional. Reinhart however shows that the CF mechanism can treat such effects of WH in situ in a similar fashion to the treatment of indicative indefinites that was reviewed above. As for definite NPs, because of their uniqueness requirement it is not easy to test their scopal behavior. I refer the reader to Winter (2001a:ch.4) for other interpretative effects with definites that are accounted for by their CF interpretation.

Crucially, we have seen above that all these kinds of NPs - simple singular/plural (in)definites and WH elements - also lead to functional readings (cf. (1), (3) and (9)-(11)). Conversely, NPs as in (12)-(14) do not give rise to wide scope readings beyond islands, and therefore they do not require the $\mathrm{CF}$ analysis. Consider for instance the contrast between at least one in the following sentence and some in (15) above.

(20) If at least one girl (I know) arrives to the party then John will be happy.

This sentence does not have the reading that is paraphrased below.

(21) There is at least one girl I know $x$ such that if $x$ arrives to the party then John will be happy.

Similar cases where WS interpretations are unavailable can also be illustrated for the other NPs in (12)-(14). This distinction between NPs where the CF analysis is available and other NPs where it is unavailable, is addressed by the syntacticsemantic mechanism that is proposed in Winter (2000, 2001a:ch.4), and I will not discuss it further here.

\subsection{General Skolem functions}

It is interesting to note that, quite independently of the scope of indefinites that have occupied semantic theories in recent years, some earlier works had proposed to use general Skolem functions (SFs) for capturing other semantic effects with indefinites and interrogatives. Intuitively, we can think of an $n$-ary Skolem function as a choice function with $n$ parameters. While a choice function $f$ maps any non-empty set $X$ to a member of $X$, a general Skolem function $f_{n}$ of arity $n$ maps such a set $X$, together with tuple of $n$ parameters $\left\langle x_{1}, \ldots, x_{n}\right\rangle$, to a member of $X$. Since we want to treat parameters in such tuples as "free variables" in Jacobson's variable-free framework, we assume that the input to a Skolem function is an $n$-place function that has the power-set $\wp(E)$ as its range. A variable-free Skolem function modifies such a function to a function that has $E$ as its range. Formally: 
Definition 2 Let $E$ and $A$ be non-empty sets. A function $f$ from $(\wp(E))^{A}$ to $E^{A}$ is a Skolem function (of arity $\geq 1$ ) iff for every function $g \in(\wp(E))^{A}$, for every $x \in A$ s.t. $g(x) \neq \emptyset:(f(g))(x) \in g(x)$. If $A$ is a Cartesian product of arity $n \geq 1$ then we say that $f$ is a Skolem function of arity $n$.

In a purely functional type-theoretical format, the domain $A$ of the functions $g$ and $f(g)$ cannot be a Cartesian product. However, Currying allows us to simulate product domains using functions and generalize the notion of Skolem functions. SFs of arity $n \geq 0$ are defined as objects of the following type scheme:

$$
\left(\tau_{1}\left(\ldots\left(\tau_{n}(\tau t)\right) \ldots\right)\right)\left(\tau_{1}\left(\ldots\left(\tau_{n} \tau\right) \ldots\right)\right) .
$$

In this type scheme, the argument of an SF is a function of type $\tau_{1}\left(\ldots\left(\tau_{n}(\tau t)\right) \ldots\right)$, which is isomorphic to a function from the Cartesian product $D_{\tau_{1}} \times \ldots \times D_{\tau_{n}}$ to the sets of $\tau$-type elements. An SF maps such a function to a function of type $\tau_{1}\left(\ldots\left(\tau_{n} \tau\right) \ldots\right)$, which is isomorphic to a function from the Cartesian product $D_{\tau_{1}} \times$ $\ldots \times D_{\tau_{n}}$ to $\tau$-type elements.

The set $S K^{n}$ of general Skolem functions of this type is defined as follows.

$$
\begin{aligned}
& S K^{n} \stackrel{\text { def }}{=} \lambda f . \forall g_{\tau_{1}\left(\ldots\left(\tau_{n}(\tau t)\right) \ldots\right)} \forall x_{1} \ldots \forall x_{n} \\
& \quad\left[g\left(x_{1}\right) \ldots\left(x_{n}\right) \neq \emptyset \rightarrow\left(g\left(x_{1}\right) \ldots\left(x_{n}\right)\right)\left((f(g))\left(x_{1}\right) \ldots\left(x_{n}\right)\right)\right]
\end{aligned}
$$

Convention: We usually assume that $\tau_{1}=\ldots=\tau_{n}=\tau$, and say that a function in $S K^{\tau, n}$ is a Skolem function (of arity $n$ ) over type $\tau$. We often write ' $S K^{n}$ ' instead of ' $S K^{n, e}$ '. With this notation it is clear that the SF of arity 0 over type $\tau$ are simply the CFs over $\tau$.

The close relationships between the general SF treatment of WS indefinites and Jacobson's treatment of functional readings are most easily demonstrated by the following examples due to Groenendijk and Stokhof (1984:ch.3) and Hintikka (1986) respectively:

(23) Every man loves a (certain) woman - his mother.

(24) According to Freud, every man unconsciously wants to marry a certain woman - his mother.

Both Groenendijk \& Stokhof and Hintikka propose to treat such examples using an existential quantifier over SFs that takes matrix scope. In the current setting, this means that the restricting predicate that the noun woman denotes must be a two-place relation, of type $e(e t)$. This is surprisingly reminiscent of Jacobson's assumption (J4), according to which restricting predicates are systematically of a higher type. Jacobson's N operator maps the set of women to the set of ee functions that map any entity to some woman or other. Instead, now we need to map the set of women to a "parameterized set of women": an $e(e t)$ function that maps any entity to the set of women. In view of this similarity, let us use the notation ' $\mathrm{N}^{0}$ ' to denote the general operator that derives such functions from ordinary denotations of nouns. Formally, the $\mathrm{N}^{0}$ operator is defined as follows.

$$
\mathrm{N}_{(e t)(e(e t))} \stackrel{\text { def }}{=} \lambda P_{e t} \cdot \lambda x_{e} \cdot \lambda y_{e} \cdot P(y)
$$


Using SFs, we can model discourses such as (23) and (24) by existential quantification over an SF at the matrix level. Even without getting into the technical derivation of the discourse binding in these examples, it is clear that existential quantification over SFs accounts for the interpretation of such sentences, which is intuitively similar to the interpretation of copular constructions such as (3). To give an illustration of this treatment, consider the following analysis of (23).

$$
\exists f_{(e(e t))(e e)}\left[S K^{1}(f) \wedge \operatorname{every}^{\prime}\left(\operatorname{man}^{\prime}\right)\left(\mathrm{Z}\left(\text { love }^{\prime}\right)\left(f\left(\mathrm{~N}^{0}\left(\text { woman }^{\prime}\right)\right)\right)\right)\right]
$$

In words: there is an Skolem function $f$ of arity 1 , which maps the function $\mathrm{N}^{0}\left(\right.$ woman $\left.^{\prime}\right)$ - the constant function that maps every entity to the set of women to an $e e$ function $h$. This $h$ function furthermore has the property that every man is in $\mathrm{Z}\left(\right.$ love $\left.^{\prime}\right)(h)$. More simply: every man loves the woman that $h$ assigns to him. Assuming that the set of women is not empty, this is equivalent to the standard analysis of sentences like (23), with narrow scope existential quantification over $e$-type entities:

$$
\text { (27) } \forall x \in \operatorname{man}^{\prime} \exists y \in \operatorname{woman}^{\prime}\left[\operatorname{love}^{\prime}(y)(x)\right]
$$

We see that in these examples, general SFs are employed to account for narrow scope readings of indefinites that due to the anaphora have a wide scope "functional flavor". It is curious to note that Reinhart's usage of the simpler 0-arity SFs (i.e. CFs) for deriving "ordinary" wide scope readings of indefinites was discovered rather late in the development of the theory.

I will not discuss here further motivations for introducing general SFs in the treatment of indefinites, and the reader is referred to many recent works that deal with this topic, including Chierchia (2001), Kratzer (1998), Schlenker (1998), Schwartz (2001) and Winter (1998,2001a:ch.3).

\section{A unified mechanism for functional readings and wide scope indefinites}

In the previous section we have seen technical, intuitive and distributional reasons to assume that "functional readings" and "wide scope indefinites" are two phenomena that should be derived by the same mechanism. However, one link is missing between the two kinds of theories that were reviewed above. In her analysis (cf. figure 1), Jacobson assumes that the restricting predicate in NPs with functional readings ranges over functions. Consequently, in her account any NP should in principle allow quantification over functions. By contrast, the CF treatment assumes that choice functions apply to the restricting predicate, which denotes a set of "ordinary" entities of type e. Quantification over CFs - if needed at all, which Kratzer (1998) doubts - is only existential, and applies syncategorematically: independently of the syntax/semantics of the NP. The same holds of the way SFs of higher arity are used for treating scopal effects with indefinites.

The main argument of this section is that this discrepancy can be resolved by renouncing Jacobson's general polymorphic scheme of quantification over functions. The main new part of the present proposal is an operator that maps one-place 
predicates over functions, of type $(e e) t$, to binary predicates of type $e(e t)$. The operator, which is called 'RG' (for "range"), is defined as follows.

$$
\mathrm{RG} \stackrel{\text { def }}{=} \lambda F_{(e e) t} \cdot \lambda x_{e} \cdot \lambda y_{e} \cdot \exists f \in F[f(x)=y]
$$

Intuitively, if $F$ is a set of ee functions, then $\mathrm{RG}(F)$ maps each entity $x$ to the set of entities $y$ that elements of $F$ assign to it. ${ }^{4}$

To illustrate how this operator allows us to unify the two mechanisms, consider again Jacobson's analysis of sentence (3a) that is illustrated in figure 1. In the revised analysis, the denotation of the "gapped" constituent every man loves still denotes the same $(e e) t$ set of functions as in Jacobson's analysis. This is the set $F=Z^{0}\left(\right.$ love $\left.^{\prime}\right)\left(\right.$ every $\left.^{\prime}\left(\operatorname{man}^{\prime}\right)\right)$ - the set of functions that map every man to something he loves. The RG operator maps $F$ to a binary predicate - the function that maps every man to the set of things he loves, provided that every man loves something. ${ }^{5}$ Let us henceforth denote this binary relation $\mathrm{RG}(F)$ by ' $S$ '. The binary relation $S$ should be intersected with the denotation of common noun woman. This can be achieved by lifting the set woman' using the $\mathrm{N}^{0}$ operator that was defined in (25) above, and was used in the treatment of sentence (23) in (26). Consequently, the restricting predicate woman that every man loves denotes the function that maps every man to the set of women he loves. Let us refer to this binary relation $\left(\mathrm{N}^{0}(\right.$ woman' $\left.) \cap S\right)$ by the letter ' $R$ '. To the binary relation $R$ we can apply the general SF mechanism, as in (26). It is easier to see how this SF mechanism works with indefinites. Therefore, let us consider how the meaning of sentence (29) below is derived. This sentence $(=(9 a))$ is a slight variation on Jacobson's example (3a).

(29) A (certain) woman that every man loves is his mother.

The analysis of this example using SFs is given in figure 2 at the end of the article, where $E C$ stands for "existential closure" - here of an SF of arity 1.

In this analysis, the Skolem function $f$ applies to the denotation of the restricting predicate woman that every man loves: the binary relation $R$ that sends each man to the set of women he loves. Assuming that $R(x)$ is non-empty for every man $x$, any SF sends $R$ to an ee function that maps every man to one of the women he loves. The statement that is derived in figure 2 claims that one of these ee functions is the mother function, which is the desired interpretation of sentence (29).

A similar analysis applies to other copular sentences with functional readings such as $(3 a-b)$ and $(11 a-b) .{ }^{6}$ Once this general interpretative mechanism is introduced, we can also come to grips with another problem of functional interpretation: which quantifiers can appear in the relative clause of a functional NP? Consider for instance the following examples.

(30) The/A woman that $\left\{\begin{array}{l}\text { every/no man (but John) } \\ * \text { at most one man } \\ \text { ?exactly/at least one man }\end{array}\right\}$ loves is his mother. 
FunCtional REAdings AND WidE-SCOPE INDEFINITES

\begin{tabular}{|c|c|c|}
\hline Noun Phrase: & \multicolumn{2}{|c|}{ Quantifier bounded by: } \\
\hline $\begin{array}{l}\text { every student } \\
\text { no student } \\
\text { every student but no teacher } \\
\text { every student but Mary } \\
\text { no student but Mary }\end{array}$ & $\begin{array}{l}\text { student } \\
\emptyset \\
\text { student } \\
\text { student } \backslash\left\{\mathrm{m}^{\prime}\right\} \\
\left\{\mathrm{m}^{\prime}\right\}\end{array}$ & $\begin{array}{l}E \\
E \backslash \text { student }^{\prime} \\
E \backslash \text { teacher }^{\prime} \\
E \backslash\left\{\mathrm{m}^{\prime}\right\} \\
E \backslash\left(\text { student }^{\prime} \backslash\left\{\mathrm{m}^{\prime}\right\}\right)\end{array}$ \\
\hline
\end{tabular}

Table 1: Bounded quantifiers

(31) Which woman does $\left\{\begin{array}{l}\text { every/no man (but John) } \\ * \text { at most one man } \\ \text { ?exactly/at least one man }\end{array}\right\}$ love? His mother.

These examples show a contrast in acceptability of functional readings between quantifiers such as every, no etc. and quantifiers such as exactly one, at most/least one etc. The following definition introduces a notion of bounded generalized quantifiers, which, I argue, is relevant to the distinction between the class of quantifiers that support functional readings and those that do not. For this definition, recall that a generalized quantifier over a domain $E$ is a set of subsets of $E$ (cf. Keenan and Westerståhl (1996)).

Definition 3 A generalized quantifier $Q \subseteq \wp(E)$ is bounded by the sets $X$ and $Y \subseteq E$ iff $Q=\{A \subseteq E: X \subseteq A \subseteq Y\}$

In other words, a quantifier $Q$ is bounded if it contains a unique minimal set $X$ and a unique maximal set $Y$, as well as all the sets $A$ that contain $X$ and are contained in $Y$. In this case we say that $X$ and $Y$ bound $Q$. Table 1 gives some NPs that denote bounded quantifiers under their standard generalized quantifier analysis, with the sets that bound these quantifiers. Now, I propose the following hypothesis:

(32) A noun phrase $N P_{1}$ in the relative clause of a complex $N P_{2}$ allows a functional reading for $N P_{2}$ only if $N P_{1}$ is a bounded quantifier.

Of course, this is not an "if and only if" condition for functional quantification, because $\mathrm{NP}_{2}$ has to be in the class of NPs that allow functional readings to begin with, which as we have seen above is rather restricted.

To illustrate the intuitive reasoning that underlies this hypothesis, consider the following contrast, reproduced from (30) above.

(33) A woman that $\left\{\begin{array}{l}\text { no } \\ * \text { at most one }\end{array}\right\}$ man loves is his mother-in-law.

As in Jacobson's analysis, the denotation of the constituent woman that no man loves is the set of functions that send each man to a woman he does not love. Let us denote this set of functions by $F_{n o}$. Assuming that $F_{n o}$ is not empty, the relation $\mathrm{RG}\left(F_{n o}\right)$ sends every man to the set of women he does not love. Applying any Skolem function of arity 1 to $\mathrm{RG}\left(F_{n o}\right)$ gives us again a function in $F_{n o}$. The situation is quite different when we consider the determiner at most one. To see that, 
let us denote the set of functions that send at most one man to a woman he loves by $F_{\leq 1}$. Assume that the men are John and Bill and that the women are Mary and Sue. Assume further that John loves Mary and hates Sue, and that Bill loves Sue and hates Mary. Thus, the set $F_{\leq 1}$ includes the following functions $f_{1}, f_{2}$ and $f_{3}$ :

$$
\begin{array}{lll}
f_{1}: & \text { John } \mapsto \text { Mary, } & \text { Bill } \mapsto \text { Mary } \\
f_{2}: & \text { John } \mapsto \text { Sue, } & \text { Bill } \mapsto \text { Sue } \\
f_{3}: & \text { John } \mapsto \text { Sue, } & \text { Bill } \mapsto \text { Mary }
\end{array}
$$

This means that $\mathrm{RG}\left(F_{\leq 1}\right)$ sends both John and Bill to the set $\{$ Mary, Sue $\}$. Consequently, there is a Skolem function that maps $\mathrm{RG}\left(F_{\leq 1}\right)$ to the function $f_{4}$ that maps each of the two men to the woman he loves:

$$
f_{4}: \text { John } \mapsto \text { Mary, Bill } \mapsto \text { Sue }
$$

But $f_{4}$ is not in $F_{\leq 1}$. In Winter (2001b) I prove that this formal distinction between the sets of functions $F_{n o}$ (for no woman) and $F_{\leq 1}$ (for at most one woman) results from the boundedness of the first quantifier vis $\bar{a}$ vis the unboundedness of the latter. Further, I argue that this difference is the origin of the two NPs' different distributions with functional readings, because only with the bounded quantifiers it is guaranteed that only functions that were generated by the mechanism are picked up by the Skolem function. In this way Skolem functions work as choice functions of a higher order. For more technical details about this account see Winter (2001b).

Two potential challenges for the empirical hypothesis in (32) should be mentioned. One problem concerns the felicitous functional interpretation of sentences such as the following, which is based on examples due to Alexopoulou and Heycock (2001).

(34) The woman that almost every/no man loves is his mother.

The natural interpretation of the noun phrase almost every man is the following.

$$
\begin{aligned}
& \left\{A \subseteq E: 1 \leq\left|\bar{A} \cap \operatorname{man}^{\prime}\right| \leq n\right\} \\
& \text { where } n \text { is a (small) number that is determined by the context. }
\end{aligned}
$$

This quantifier in not bounded. However, sentence (34) can also be interpreted as in the rough paraphrase below.

(36) There is a (small) set of men $B$ s.t. the woman that every/no man except for the men in $B$ loves is his mother.

In other words: the "exception set" $B$ that almost quantifiers require can take here sentential scope, which allows the noun phrase almost every/no man to be interpreted as a bounded quantifier. ${ }^{7}$

Another problem for the hypothesis in (32) may come from certain facts that are pointed out by Sharvit (1999:(18)-(21)). Sharvit considers plural sentences in Hebrew that are parallel to the following English sentence. ${ }^{8}$

(37) The woman that most of the students invited was their mother.

The determiner most is unbounded, hence functional anaphora is a priori not ex- 
pected here by hypothesis (32). However, with noun phrases such as most of the students, also discourse anaphora as in the following example may appear and complicate the picture.

(38) Most of the students admire their mother. They invited her.

By contrast, discourse anaphora is unacceptable with the other quantifiers that illustrated (un)availability of functional readings in the above examples. For instance:

(39) No/at most one student hates his mother. *He invited her.

This means that the contrast we observed above between no and at most one cannot originate from different discourse anaphora potentials, and therefore it supports hypothesis (32). The "functional reading" in Sharvit's example (37) may result from discourse anaphora and therefore it does not seriously challenge this hypothesis. For recent works. that deal with discourse anaphora using a mechanism of functional quantification see Steedman (1999) and Peregrin and Von Heusinger (2001).

\section{A note on Jacobson (these proceedings)}

In her article, Jacobson shows motivation for an operator that is strikingly similar to the ' $\mathrm{RG}$ ' operator, although her motivation comes from different phenomena than the ones that were addressed throughout the present paper. The examples that motivate Jacobson's novel operator are all similar to the following one.

(40) The woman he loves that every/no man invited (to his wedding) is his mother.

Jacobson (1994) lifts the denotation of the noun woman to a set of ee functions. However, in her variable-free treatment of anaphora, the denotation of the relative he loves, which should be intersected with the denotation of woman, is a binary relation. Hence, Jacobson proposes to shift this binary relation into a set of functions using the following operator, which she calls ' $\mathrm{m}$ '.

$$
\mathbf{m}_{(e(e t))((e e) t)} \stackrel{\text { def }}{=} \lambda R_{e(e t)} \cdot \lambda f_{e e} \cdot \forall x_{e} \cdot R(x)(f(x))
$$

In many categorial accounts of extraction, the relative he loves denotes the following relation:

(42) he loves' $=\lambda x \cdot \lambda y \cdot \operatorname{love}^{\prime}(y)(x)$

Intersection with the functional denotation of the noun woman leads to the following analysis:

(43) $\mathrm{N}\left(\right.$ woman $\left.^{\prime}\right) \cap \mathbf{m}($ he loves' $)=$ $\left(\lambda f_{e e} \cdot \forall z\left[\operatorname{woman}^{\prime}(f(z))\right]\right) \cap\left(\lambda f_{e e} \cdot \forall u\left[\operatorname{love}^{\prime}(f(u))(u)\right]\right)=$ $\lambda f_{e e} \cdot \forall z\left[\operatorname{woman}^{\prime}(f(z))\right] \wedge \forall u\left[\operatorname{love}^{\prime}(f(u))(u)\right]$

In words, this is the set of ee functions that send every $x$ to a woman that $x$ loves. Once this set of functions is derived, the rest of the analysis of sentence (40) is 
identical to the analysis of sentence (3a) in figure 1 .

In the present proposal, since the noun woman is anyway lifted to a binary relation using the $\mathrm{N}^{0}$ operator, there is no need to use further shiftings, and the analysis of the relative nominal woman he loves is as follows, which gives the same result as in Jacobson's analysis.

$$
\begin{aligned}
& \mathrm{N}^{0}\left(\operatorname{woman}^{\prime}\right) \cap\left(\text { he } \text { loves }^{\prime}\right)= \\
& \left(\lambda x \cdot \lambda y \cdot \operatorname{woman}^{\prime}(y)\right) \cap\left(\lambda x \cdot \lambda y \cdot \operatorname{love}^{\prime}(y)(x)\right)= \\
& \lambda x \cdot \lambda y \cdot \operatorname{woman}^{\prime}(y) \wedge \operatorname{love}^{\prime}(y)(x)
\end{aligned}
$$

We conclude, in agreement with Jacobson, that her strategy is in a sense the inverse of the present proposal. Jacobson shif ts relatives from binary relations into sets of functions, while in this paper we used the opposite direction - from sets of functions to binary relations - using the $R G$ operator.

I find it quite notable that the RG mechanism, which was developed for entirely different reasons than Jacobson's $m$ operator, also handles the problem that motivated Jacobson's approach. It is also quite likely that Jacobson's mechanism can be adapted to handle at least some of the problems that were treated in the present paper.

\section{Conclusions}

We developed a technique that uses Skolem functions uniformly to treat both widescope indefinites and functional readings. This mechanism led us to an hypothesis that may explain the distribution of various quantifiers with functional questions and copular sentences. The study of functional quantification is surely far from its end. However, I believe that further synthesis of techniques from variable-free semantics, categorial logic, Skolem functions and generalized quantifiers into linguistic theory may lead us to significant progress in our understanding of the relevant phenomena.

\section{Endnotes}

* This research was partly supported by grant no. 1999210 ("Extensions and Implementations of Natural Logic") from the United States-Israel Binational Science Foundation (BSF), Jerusalem, Israel. The author was also partly supported by the fund for the promotion of research at the Technion, research no. 120-042, and by an NWO grant for visiting the UiL OTS of the Utrecht University in summer 2001. I am grateful to the organizers of the ESSLLI-2001 workshop on choice functions for their invitation, which initiated this work. Thanks also to Edit Doron, Nissim Francez, Carolyn Heycock, Pauline Jacobson, Tanya Reinhart and Eddy Ruys for discussion.

1. See Engdahl (1986:ch.4) and Groenendijk and Stokhof (1984:ch.3) for two classical works on this topic.

2. Jacobson's mechanism achieves this using (a modified version of) the "Geach 
Rule" and Function Composition.

3. Some authors, notably Kratzer (1998), favor a usage of CFs as "deictic" entities, without existential quantification over them. This controversy is not central for the purposes of this paper, and readers may easily modify the analyses below to the "deictic" version of CFs.

4. Alternatively, we can standardly view an ee function $f$ as the binary relation $R_{f} \stackrel{\text { def }}{=}\{\langle x, y\rangle: y=f(x)\}$. Then $\mathrm{RG}(F)$ is simply the union of the relations that correspond to functions in $F: R G(F)=\cup_{f \in F} R_{f}$. I thank Nissim Francez for pointing this out to me.

5. If there is a man who does not love anything, then $F$ is empty and consequently $\mathrm{RG}(F)$ sends everything to the empty set.

6. The full details on the analysis of these examples appear in Winter (2001b).

7. This is not always the case with almost quantifiers. For instance, a sentence such as every Frenchman admires almost every actress, is compatible with a situation where one Frenchman admires every actress but Brigitte Bardot, while another Frenchman admires every actress but Isabelle Adjani.

8. Sharvit also judges as felicitous sentences like (37) with more than two and at most two instead of most of the. However, my Hebrew informants disagree, and consider these cases significantly worse than the Hebrew parallel of (37).

\section{References}

Alexopoulou, D. and Heycock, C. (2001). Definites and indefinites in relative clauses. In Proceedings of the Workshop on Choice Functions and Natural Language Semantics. Arbeitspapier 110: FB Sprachwissenschaft. Universität Konstanz. Can be downloaded from http://ling.unikonstanz.de/pages/home/heusinger/publications/abstracts/ap110.html.

Chierchia, G. (1993). Questions with quantifiers. Natural Language Semantics, $1: 181-234$.

Chierchia, G. (2001). A puzzle about indefinites. In C. Cecchetto, G. C. and Guasti, M. T., editors, Semantic Interfaces: reference, anaphora and aspect. CSLI Publications, Stanford.

Engdahl, E. (1986). Constituent Questions: the syntax and semantics of questions with special reference to Swedish. D. Reidel, Dordrecht.

Fodor, J. D. and Sag, I. (1982). Referential and quantificational indefinites. Linguistics and Philosophy, 5:355-398.

Groenendijk, J. and Stokhof, M. (1984). Studies on the Semantics of Questions and the Pragmatics of Answers. PhD thesis, University of Amsterdam.

Hintikka, J. (1986). The semantics of a certain. Linguistic Inquiry, 17:331-336.

Jacobson, P. (1994). Binding connectivity in copular sentences. In Proceedings of Semantics and Linguistic Theory, SALT4.

Jacobson, P. (1999). Towards a variable-free semantics. Linguistics and Philosophy, 22:117-185.

Jacobson, P. (2000). Paycheck pronouns, Bach-Peters sentences, and variable-free 
semantics. Natural Language Semantics, 8:77-155.

Jacobson, P. (2002). Direct compositionality and variable-free semantics: the case of binding into heads. These proceedings.

Keenan, E. and Westerståhl, D. (1996). Generalized quantifiers in linguistics and logic. In van Benthem, J. and ter Meulen, A., editors, Handbook of Logic and Language. Elsevier, Amsterdam.

Kratzer, A. (1998). Scope or pseudoscope? Are there wide scope indefinites? In Rothstein, S., editor, Events and Grammar. Kluwer, Dordrecht.

Peregrin, J. and von Heusinger, K. (2001). Dynamic semantics with choice functions. In Kamp, H. and Partee, B., editors, Context Dependence in the Analysis of Linguistic Meaning. Elsevier Science, Amsterdam. in press.

Reinhart, T. (1992). Wh-in-situ: an apparent paradox. In Proceedings of the 8th Amsterdam Colloquium. University of Amsterdam, Institute for Logic, Language and Computation.

Reinhart, T. (1997). Quantifier scope: how labor is divided between QR and choice functions. Linguistics and Philosophy, 20:335-397.

Schlenker, P. (1998). A note on Skolem functions and the scope of indefinites. Unpublished ms., MIT.

Schwartz, B. (2001). Two kinds of long-distance indefinites. In Proceedings of the 13th Amsterdam Colloquium. University of Amsterdam, Institute for Logic, Language and Computation.

Sharvit, Y. (1999). Functional relative clauses. Linguistics and Philosophy, 22:447478.

Steedman, M. (1999). Altemating quantifier scope in CCG. In Proceedings of the 37th annual meeting of the Association for Computational Linguistics (ACL).

Winter, Y. (1998). Flexible Boolean Semantics: coordination, plurality and scope in natural language. $\mathrm{PhD}$ thesis, Utrecht University.

Winter, Y. (2000). DP structure and flexible semantics. In Proceedings of the 30th conference of the Northeast Linguistic Society, NELS30.

Winter, Y. (2001a). Flexibility Principles in Boolean Semantics: coordination, plurality and scope in natural language. MIT Press, Cambridge, Massachusetts.

Winter, Y. (2001b). Functional quantification. In Proceedings of the Workshop on Choice Functions and Natural Language Semantics. Arbeitspapier 110: FB Sprachwissenschaft. Universität Konstanz. Can be downloaded from http://ling.unikonstanz.de/pages/home/heusinger/publications/abstracts/ap110.html.

Computer Science

Technion - Israel Institute of Technology

Haifa 32000

Israel

E-mail: winter@cs.technion.ac.il

WWW: http://www.cs.technion.ac.il/ winter 

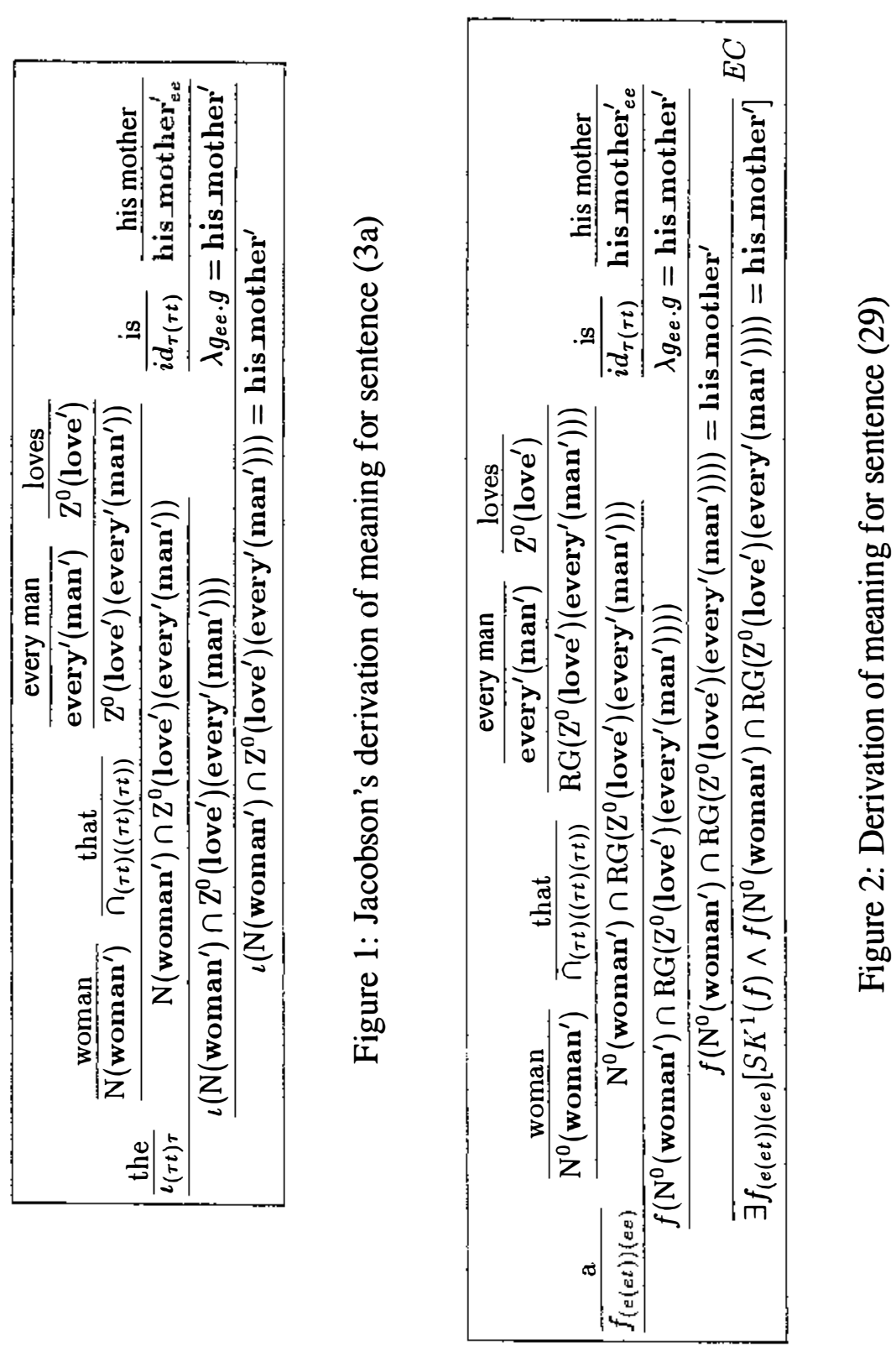